\title{
NOVIKOV-BETTI NUMBERS AND THE FUNDAMENTAL GROUP
}

\author{
M. FARBER AND D. SCHÜTZ
}

Theorem 1. Let $X$ be a connected finite polyhedron and let $\xi \in H^{1}(X ; \mathbf{R})$ be a nonzero cohomology class. If the first Novikov-Betti number $b_{1}(\xi)$ is nonzero, $b_{1}(\xi)>0$, then $\pi_{1}(X)$ contains a nonabelian free subgroup.

This result may appear striking as the Novikov-Betti numbers carry "abelian" information about $X$. We refer to [4, 3] for the definition of the Novikov-Betti numbers; an explicit definition will also be given below in the proof of Theorem 1

An alternative description of $b_{i}(\xi)$ uses homology of complex flat line bundles. Consider the variety $\mathcal{V}_{\xi}$ of all complex flat line bundles $L$ over $X$ having the following property: $L$ has trivial monodromy along any loop $\gamma$ in $X$ assuming that $\langle\xi,[\gamma]\rangle=0$. It is easy to see that (a) $\mathcal{V}_{\xi}$ is an algebraic variety isomorphic to $\left(\mathbf{C}^{*}\right)^{r}$ for some integer $r$ and (b) the dimension $\operatorname{dim} H_{i}(X ; L)$ is independent of $L$ assuming that $L \in \mathcal{V}_{\xi}$ is generic, see [3], Theorem 1.50. The number $\operatorname{dim} H_{i}(X ; L)$ for a generic $L \in \mathcal{V}_{\xi}$ coincides with the Novikov-Betti number $b_{i}(\xi)$.

The proof of Theorem 1 given below is based on the results of R. Bieri, W. Neumann, R. Strebel [1] and J.-Cl. Sikorav 6].

Theorem 1 implies the following vanishing result:

Corollary 1. Let $X$ be a connected finite polyhedron having an amenable fundamental group. Then the first Novikov-Betti number vanishes $b_{1}(\xi)=0$ for any $\xi \neq 0 \in H^{1}(X ; \mathbf{R})$.

Corollary 1 follows from Theorem 1 as an amenable group contains no nonabelian free subgroups. As another simple corollary of Theorem 1 we mention the next statement:

Corollary 2. Assume that $X$ is a connected finite two-dimensional polyhedron. If the Euler characteristic of $X$ is negative $\chi(X)<0$ then $\pi_{1}(X)$ contains a nonabelian free subgroup.

Proof. The first Betti number $b_{1}(X)$ is positive as follows from $\chi(X)<0$. Hence there exists a nonzero cohomology class $\xi \in H^{1}(X ; \mathbf{R})$. Then

$$
\chi(X)=b_{2}(\xi)-b_{1}(\xi),
$$

see Proposition 1.40 in $\left[3\right.$. Here we use that $b_{0}(\xi)=0$ for $\xi \neq 0$, see Corollary 1.33 in 3. The inequality $\chi(X)<0$ together with (11) imply that $b_{1}(\xi)>0$. Theorem 1 now states that $\pi_{1}(X)$ contains a nonabelian free subgroup.

Corollary 2 is known, it was obtain by N.S. Romanovskii, see [5]. It can be equivalently expressed algebraically as follows:

Corollary 3. Any discrete group $G$ of deficiency greater than 1 contains a nonabelian free subgroup. 
The authors thank Jonathan Hillman for referring to [5].

Proof of Theorem [1. Consider the following diagram of rings and ring homomorphisms:

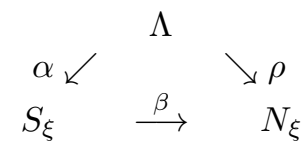

Here $\Lambda=\mathbf{Z}[\pi]$ is the group ring of the fundamental group of $X$ which we denote $\pi=\pi_{1}\left(X, x_{0}\right)$.

The ring $N_{\xi}$ is the Novikov ring which is defined as follows. View the class $\xi$ as a group homomorphism $\xi: \pi \rightarrow \mathbf{Z}$ and let $H$ denotes the factorgroup $\pi / \operatorname{Ker}(\xi)$. It is a finitely generated free abelian group. The usual group ring $\mathbf{Z}[H]$ consists of finite sums of the form $\sum a_{j} h_{j}$ with $a_{j} \in \mathbf{Z}$ and $h_{j} \in H$; it coincides with the Laurent polynomial ring in $\operatorname{rk} H$ variables. The Novikov ring $N_{\xi}$ is a completion of $\mathbf{Z}[H]$; its elements are infinite sums $\sum_{j=1}^{\infty} a_{j} h_{j}$ with $a_{j} \in \mathbf{Z}$ and $h_{j} \in H$ such that the sequence of evaluation $\xi\left(h_{j}\right)$ tends to $-\infty$. In other words, $N_{\xi}$ is the ring of Laurent power series in $\operatorname{rk} H$ variables where the terms of the series go to infinity in the direction specified by the class $\xi$.

The ring $S_{\xi}$ is the Novikov - Sikorav completion of the group ring $\Lambda=\mathbf{Z}[\pi]$; it was originally introduced by J.-Cl. Sikorav [6]. Elements of $S_{\xi}$ are infinite sums of the form $\sum_{j=1}^{\infty} a_{j} g_{j}$ where $a_{j} \in \mathbf{Z}, g_{j} \in \pi$ and $\xi\left(g_{j}\right) \rightarrow-\infty$.

Diagram (2) includes the obvious rings homomorphisms $\alpha, \beta, \rho$ and is commutative.

Let $X$ be a finite connected polyhedron with fundamental group $\pi$. Consider the universal covering $\tilde{X} \rightarrow X$. The cellular chain complex $C_{*}(\tilde{X})$ is a complex of finitely generated free left $\Lambda$-modules. Since $N_{\xi}$ is a commutative ring and a principal ideal domain (see [3], Lemma 1.10) the homology $H_{i}\left(N_{\xi} \otimes_{\Lambda} C_{*}(\tilde{X})\right)$ is a finitely generated $N_{\xi}$-module and its rank (over $N_{\xi}$ ) equals the Novikov-Betti number $b_{i}(\xi)$, see [3], $\S 1.5$.

Our first goal is to show that the assumption $b_{1}(\xi) \neq 0$ implies

$$
H_{1}\left(S_{\xi} \otimes_{\Lambda} C_{*}(\tilde{X})\right) \neq 0 .
$$

Indeed, note that $H_{0}\left(S_{\xi} \otimes_{\Lambda} C_{*}(\tilde{X})\right)=0$ (as we assume that $\xi \neq 0$ ). Hence, using the Künneth spectral sequence we find that

$$
\begin{aligned}
H_{1}\left(N_{\xi} \otimes_{\Lambda} C_{*}(\tilde{X})\right) & =H_{1}\left(N_{\xi} \otimes_{S_{\xi}}\left(S_{\xi} \otimes_{\Lambda} C_{*}(\tilde{X})\right)\right) \\
& =N_{\xi} \otimes_{S_{\xi}} H_{1}\left(S_{\xi} \otimes_{\Lambda} C_{*}(\tilde{X})\right) .
\end{aligned}
$$

Hence $b_{1}(\xi) \neq 0$ implies the nonvanishing of $H_{1}\left(N_{\xi} \otimes_{\Lambda} C_{*}(\tilde{X})\right)$ which via (4) gives (3).

Note that $b_{1}(\xi)=b_{1}(-\xi)$ (see Corollary 1.31 in $[\underline{3}$ ) and hence the nonvanishing of the Novikov-Betti number $b_{1}(\xi)$ implies the nonvanishing of the Novikov - Sikorav homology $H_{1}\left(S_{\xi} \otimes_{\Lambda} C_{*}(\tilde{X})\right)$ for both cohomology classes $\xi$ and $-\xi$.

R. Bieri, W. Neumann and R. Strebel [1] defined a geometric invariant of discrete groups. It can be viewed as a subset $\Sigma$ of the space of nonzero cohomology classes $\Sigma \subset H^{1}(X ; \mathbf{R})-\{0\}$. A theorem of Jean-Claude Sikorav (see [6], page 86 or [2]) states that for a nonzero cohomology class $\xi \in H^{1}(X ; \mathbf{R})$ the following conditions are equivalent: (a) $H_{1}\left(S_{\xi} \otimes_{\Lambda} C_{*}(\tilde{X})\right)=0$ and (b) $-\xi \in \Sigma$. Hence, as explained 
above, $b_{1}(\xi) \neq 0$ implies that $\xi \notin \Sigma$ and $-\xi \notin \Sigma$. Now we apply Theorem $\mathrm{C}$ from [1] which states that the union of $\Sigma$ and $-\Sigma$ equals $H^{1}(X ; \mathbf{R})-\{0\}$ assuming that $\pi_{1}(X)$ has no non-abelian free subgroups. Since in our case neither $\xi \in \Sigma$ nor $-\xi \in \Sigma$ we conclude that $\pi_{1}(X)$ must contain a non-abelian free subgroup.

Next we mention an example showing that a space with nonvanishing Novikov torsion number $q_{1}(\xi)$ may have an amenable fundamental group. In other words, Theorem 1becomes false if one replaces the assumption $b_{1}(\xi) \neq 0$ by the assumption $q_{1}(\xi) \neq 0$. Example 1.49 in $\left[3\right.$ gives a two-dimensional polyhedron with $b_{1}(\xi)=0$ and $q_{1}(\xi)=1$ for some nonzero $\xi \in H^{1}(X ; \mathbf{R})$. The fundamental group of $X$ is a Baumslag-Solitar group $G=\left|a, b ; a b a^{-1}=b^{2}\right|$. The later group appears in the exact sequence $0 \rightarrow \mathbf{Z}\left[\frac{1}{2}\right] \rightarrow G \rightarrow \mathbf{Z} \rightarrow 0$ (see [3], page 30); hence $G$ is amenable.

\section{REFERENCES}

[1] R. Bieri, W. Neumann, R. Strebel, A geometric invariant of discrete groups, Invent. Math. 90(1987), $451-477$.

[2] R. Bieri, The geometric invariants of a group; a survey with emphasis on the homotopical approach, Geometric group theory, pp. 24 - 36, Camb. Univ. Press, 1993.

[3] M. Farber, Topology of closed one-forms. Mathematical Surveys and Monographs, 108. American Mathematical Society, Providence, RI, 2004.

[4] S. Novikov, Multi-valued functions and functionals. An analogue of Morse theory. Soviet. Math. Doklady, 24(1981), 222-226.

[5] N.S. Romanovskii, Free subgroups of finitely presented groups, Algebra and Logic 16(1977), no. $1,62-68$

[6] J.-Cl. Sikorav, Thèse, Université Paris-Sud, 1987.

Department of Mathematics, University of Durham, Durham DH1 3LE, UK

E-mail address: Michael.Farber@durham.ac.uk

E-mail address: Dirk.Schuetz@durham.ac.uk 